\title{
Efectos del vendaje neuromuscular sobre la flexibilidad del raquis lumbar
}

\author{
Labrador-Cerrato AM. ${ }^{1 a}$, Ortega Sánchez-Diezma P. ${ }^{2 a}$, Lanzas Melendo G. ${ }^{3 a}$, Gutiérrez-Ortega C. ${ }^{4 b}$ \\ Sanid. mil. 2015; 71 (1): 15-21; ISSN: 1887-8571
}

\begin{abstract}
RESUMEN
Introducción: El vendaje neuromuscular es una técnica que produce una estimulación muy selectiva sobre la piel a través de la aplicación de unas vendas elásticas especiales con el fin de lograr cambios propioceptivos, aumento o inhibición del tono muscular y mitigación de algias, entre otros. Objetivos: Comprobar si la aplicación del vendaje neuromuscular permite aumentar la flexión del raquis lumbar comparándola con otras técnicas de vendaje placebo (esparadrapo rígido convencional; Omniplaste $\left.{ }^{\circledR}-E\right)$; observar la concordancia entre la prueba sit-and-reach y el test de Schober en la valoración de la ganancia de flexión lumbar. Material y métodos: Estudio piloto experimental a triple ciego. Se distribuyeron aleatoriamente 45 sujetos sanos de 20-55 años en tres grupos: 1) esparadrapo convencional; 2) Omniplaste ${ }^{\circledR}$-E; 3) vendaje neuromuscular. En todos los participantes se evaluó la flexión del raquis lumbar mediante la prueba sit-and-reach y el test de Schober antes y después de la intervención siguiendo el mismo protocolo. Resultados: Considerado un intervalo de confianza del 95\% y grado de significación estadística $\mathrm{p}<0,05$ en todos los casos, se obtuvo un incremento estadísticamente significativo de la flexión lumbar en todos los grupos según la prueba sit-and-reach, que fue mayor en el grupo del vendaje neuromuscular $(1,5 \mathrm{~cm}$ de mediana; $\mathrm{p}=0,011)$. Según el test de Schober, solamente el vendaje neuromuscular se mostró eficiente $(\mathrm{p}<0,001)$, incrementándose el valor basal en un 6,25\% (1 cm de mediana). Conclusiones: La aplicación del vendaje neuromuscular sobre la columna lumbar mejora la flexión lumbar respecto a técnicas placebo a partir de los resultados obtenidos mediante el test de Schober, así como una mayor flexión global del tronco que estas técnicas según la prueba sit-and-reach. El test de Schober parece ser más fiable y preciso que la prueba sit-and-reach para estudios de este tipo.
\end{abstract}

PALABRAS CLAVE: Vendaje neuromuscular; Flexibilidad; Región lumbosacra; Kinesiotape; Sit-and-reach; Schober.

\section{Effects of kinesio taping on lumbar rachis flexibility}

SUMMARY: Introduction: Kinesio taping is a technique that produces a very selective stimulation through skin by means of specific elastic strips application aiming to achieve proprioceptive changes, increase or inhibition of muscle activity and pain mitigation, among others. Aims: The present work was focused on checking whether kinesio taping increases lumbar rachis flexion compared with other placebo taping techniques such as conventional tape or Omniplaste ${ }^{\circledR}-E$ elastic tape. Furthermore, it was led to observe the correspondence between the sit-and-reach and Schober tests when assessing the increase of lumbar flexion. Material and methods: Experimental, triple-blind randomized controlled pilot trial, where 45 healthy 20-55 year-aged participants were randomly distributed into three groups: 1) conventional taping; 2) Omniplaste ${ }^{\circledR}-E$; 3) kinesio taping. Flexion of lumbar rachis was evaluated using both sit-and-reach and Schober tests before and after the intervention following the same procedure in all subjects. Results: Considered $95 \%$ as confidence interval and level of significant difference of $\mathrm{p}<0.05$ in all cases, a statistically significant increase on lumbar flexion was obtained in all groups by sit-and-reach test measure procedures, even higher in kinesio taping group ( $1.5 \mathrm{~cm}$ median; $\mathrm{p}=0.011)$. Taking into account Schober test values, only kinesio taping group was efficient $(\mathrm{p}<0.001)$, increasing basal measure up to $6.25 \%$ ( $1 \mathrm{~cm}$ median). Conclusions: Application of kinesio taping on lumbar rachis helps to increase lumbar flexion better than some placebo techniques from results obtained by Schober test procedures. It was also observed that kinesio taping achieves a higher global trunk flexion respecting such techniques by sitand-reach test. Schober test seems to be more accurate and reliable than sit-and-reach test for this type of studies.

KEYWORDS: Ahletic tape; Lumbosacral region; Joint flexibility; Kinesiotape; Musculoskeletal system; Physical therapy modalities.

${ }^{1}$ Graduado en Fisioterapia.

${ }^{2}$ Diplomada en Fisioterapia (Máster Oficial)

${ }^{3}$ T.Col Médico.

${ }^{4}$ Doctor en Medicina.

a Servicio de Rehabilitación. ${ }^{\mathrm{b}}$ Servicio de Medicina Preventiva.

Hospital Central de la Defensa. Madrid. España.

Dirección para correspondencia: Ángel M Labrador-Cerrato. Servicio de Rehabilitación. Hospital Central de la Defensa. Glorieta del Ejército 1. 28047 Madrid, España.Tfno: (+34) 914222175. Fax: (+34) 914222568. alabcer@oc.mde.es

Recibido: 11 de marzo de 2014

Aceptado: 3 de septiembre de 2014

\section{INTRODUCCIÓN}

\section{Antecedentes históricos}

El vendaje neuromuscular es una técnica especial de vendaje creada por Kenzo $\mathrm{Kase}^{1}$, un médico quiropráctico japonés que tras varios años de pruebas, investigación y ensayos clínicos, desarrolló unas cintas elásticas adhesivas especiales que constituirían la base del vendaje neuromuscular, creado a mediados de los años 70; sin embargo, éste no fue fundado oficialmente hasta 
finales de esa década. Dicho método también ha sido conocido como KinesioTaping, Kinesio Tape, Vendaje Neuromuscular, Medical Taping Concept, así como otros muchos términos, debido en parte a distintas traducciones al inglés y también a los nombres de las numerosas marcas empresariales que comercializan las vendas elásticas utilizadas, contribuyendo así a una denominación bastante difusa. En este trabajo, nos referiremos a esta técnica como Vendaje Neuromuscular (VNM).

El VNM apareció a nivel internacional en 1988 durante los Juegos Olímpicos de Seúl en varios deportistas japoneses y, desde entonces, el método ha crecido paulatinamente llegando a ser un tratamiento a nivel mundial, en gran medida publicitado y potenciado por el deporte de élite, entrando en Europa a partir del año 2000 en el ámbito de la Fisioterapia.

\section{Situación actual}

La progresión de este método ha provocado que se haya extendido hasta estar presente en más de 27 países en la actualidad. Es habitual observarlo en el mundo del deporte, pero también se ha afianzado en los tratamientos de personas no deportistas, en Fisioterapia Neurológica, en la rehabilitación de procesos traumatológicos y Fisioterapia en general. Actualmente se investiga en nuevos campos de aplicación, lográndose avances en Terapia Ocupacional o incluso en la rehabilitación de animales (caballos de carreras), de modo que en la práctica es una herramienta habitual de trabajo a la que debe prestarse atención gracias a su éxito y buena aceptación.

\section{Justificación de la presente investigación}

El VNM pretende causar una estimulación muy selectiva a través de la aplicación de unas vendas elásticas especiales sobre la piel, con el fin de lograr ciertos cambios propioceptivos, un aumento o inhibición del tono muscular, y mitigación de procesos álgicos o inflamatorios, entre otros ${ }^{2,3}$. El vendaje empleado posee unas propiedades específicas similares a las de la piel tales como el peso o la densidad ${ }^{1,4}$, destacando también las referentes a su comportamiento mecánico. Este hecho permite una estimulación directa y sumamente eficaz al proporcionar información a los nociceptores ligamentosos para eliminar el bucle dolor-rigidez $^{5}$, y a los mecanorreceptores (junto a los husos neuromusculares y órganos tendinosos de Golgi) para normalizar así el estado de activación muscular ${ }^{6,7}$.

Según Kapandji, la flexión lumbar con enderezamiento o rectificación de la lordosis llega hasta unos $40^{\circ}$ de amplitud como valor promedio ${ }^{8}$, variando significativamente según la edad del sujeto, y que responde a la suma del movimiento entre las vértebras L1, L2, L3, L4 y L5; se encuentra limitado por varios ligamentos intervertebrales, fascia dorsolumbar y la musculatura paravertebral lumbar.

El presente trabajo parte desde un punto de vista no propiamente terapéutico, sino que se orienta a un aspecto biomecánico o postural, de modo que su primer objetivo consiste en comprobar que con una cierta colocación del VNM se puede aumentar la flexión del raquis lumbar en ausencia de dolor ${ }^{9}$. La falta de flexibilidad debida a un acortamiento de tipo miofascial, rigidez articular o hipertonía muscular condiciona que la flexión lumbar pueda verse limitada, aumentando la frecuencia de patologías secundarias en el raquis. La columna lumbar destaca como uno de los motivos más habituales de consulta en Aparato Locomotor, sobre todo cuando presenta hiperlordosis (puede producir otras patologías secundarias en el resto del raquis).

En segundo lugar, esta investigación trata de valorar si el aumento de la flexión lumbar se debe a un efecto placebo ocasionado por tener simplemente un vendaje aplicado sobre la piel (esparadrapo convencional o Omniplaste $^{\circledR}-E$ ), o al efecto que el VNM provoca.

En tercer lugar, se pretende observar la concordancia entre la prueba sit-and-reach (SAR) y el test de Schober para valorar la ganancia de la flexión lumbar. Aunque la prueba SAR está validada, sus niveles de fiabilidad son cuestionables al ser una prueba que incluye también a todos los miembros y el raquis, lo que puede distorsionar los resultados. No obstante, se emplea en trabajos sobre flexibilidad lumbar y, por tanto, se ha querido emplear a fin de facilitar la comparación de resultados, si bien no es la prueba de elección de los autores del presente estudio.

Por esta razón, se ha aplicado el test de Schober para conocer con exactitud lo que sucede exclusivamente en la columna lumbar. Más adelante se detallará cómo se han llevado a cabo ambas pruebas de medición.

Por último, debido a la escasez de artículos concluyentes relacionados con los objetivos del presente trabajo ${ }^{10}$, la investigación actual se basó en un estudio piloto con 45 individuos sanos con el propósito de poder estimar el tamaño muestral necesario para considerar el estudio como concluyente, teniendo en cuenta además el posible sesgo de edad y sexo.

\section{MATERIAL Y MÉTODOS}

\section{Participantes, material y diseño del estudio}

El estudio se dirigió a trabajadores del Hospital Central de la Defensa (Madrid), incluyendo a personas de entre 20 y 55 años de edad, de ambos sexos y cualquier categoría laboral, asintomáticos, excluyéndose los que hubieran sido intervenidos quirúrgicamente en el raquis, sufrieran alguna lesión en los músculos isquiosurales, zona lumbar, cadera, rodillas o gemelos, o estuvieran tomando actualmente medicación analgésica o antiinflamatoria.

Se realizó un estudio piloto experimental a triple ciego que garantizó el enmascaramiento de las técnicas aplicadas, donde la persona que llevó a cabo las mediciones ignoraba la intervención aplicada, y quien realizó el análisis estadístico dispuso exclusivamente de los datos numéricos (cada técnica de vendaje se trató como «Terapia 1, 2 ó 3»). Ningún sujeto supo tampoco qué intervención de las tres se le iba a aplicar.

Se llevó a cabo un muestreo consecutivo no probabilístico durante los meses de Febrero a Abril de 2013, reclutando a los sujetos conforme acudían voluntariamente para ser tratados, tras leer los carteles distribuidos por los distintos Servicios del centro en los que se les invitaba al estudio. Finalmente fueron incluidos los 45 participantes que superaron los criterios de in- 


\section{Efectos del vendaje neuromuscular sobre la flexibilidad del raquis lumbar}

clusión y exclusión, distribuidos aleatoriamente en 3 grupos de 15 individuos cada uno, paso previo a la realización de las mediciones iniciales. Además, fueron convenientemente informados sobre el estudio y dieron su consentimiento para tratar sus datos personales de manera confidencial, incluyendo el Servicio en el que trabajaban, edad y teléfono de contacto. Se tuvo en cuenta el sexo y la edad para el estudio estadístico posterior, pero esto no influyó a la hora de modificar la aplicación de las intervenciones.

La distribución de los participantes en los tres grupos se detalla en la Tabla 1.

Tabla 1. Características de los sujetos asignados a los 3 grupos de técnicas.

\begin{tabular}{|lllll|}
\hline & 1-Esparadrapo & 2- Omniplaste ${ }^{\circledR}-E$ & 3-VNM & p \\
\hline Sujetos & $\mathrm{n}=15$ & $\mathrm{n}=15$ & $\mathrm{n}=15$ & \\
Sexo (varón/mujer) & $7 / 8$ & $9 / 6$ & $5 / 10$ & 0,343 (c2 Pearson) \\
Edad (años) & $47(8)$ & $43(11)$ & $46(10)$ & 0,452 (ANOVA) \\
\hline
\end{tabular}

La edad media de la muestra fue de 45,4 años, con una máxima de 55 y mínima de 21 años, y con un 46,7\% de hombres y $53,3 \%$ de mujeres. La aleatorización garantizó la homogeneidad de los 3 grupos en cuanto a las variables del sexo y la edad.

En cuanto al material de medición necesario para la prueba SAR, se empleó un cajón con una cinta métrica adherida a su tapa superior (ver Figura 1) con unas medidas de $27 \times 39 \times 75$ -alto/ancho/largo- (en cm; la tapa superior sobresalía 31,4 cm), así como otra cinta métrica convencional para el test de Schober.

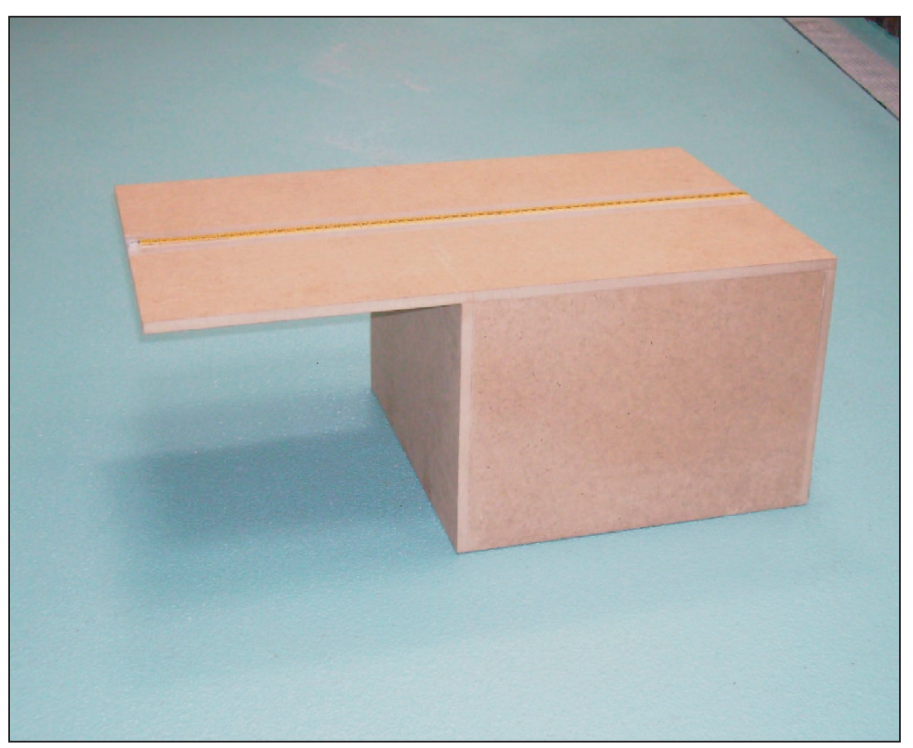

Figura 1. Cajón empleado para la prueba sit-and-reach.

Todas las vendas utilizadas tienen una anchura de $5 \mathrm{~cm}$. El esparadrapo utilizado pertenece a la marca HARTMANN, modelo Omniplast ${ }^{\circledR}$ White. La venda elástica adhesiva pertenece a la marca $B S N$ MEDICAL, modelo Omniplaste ${ }^{\circledR}$-E. El VNM pertenece a la marca $B S N$ MEDICAL GmbH, modelo Leukotape ${ }^{\circledR} \mathrm{K}$.

\section{METODOLOGÍA}

\section{Medición de la flexibilidad lumbar}

La dificultad principal de la medición de la flexión lumbar reside en que casi ninguna prueba es capaz de aislar la movilidad de esta zona de otros segmentos del raquis, ni tampoco de la extensibilidad de la musculatura posterior de los miembros inferiores $^{11}$. Una de las más utilizadas es la prueba $\mathrm{SAR}^{12}$, que consiste en que un sujeto sentado en el suelo con las rodillas extendidas intente realizar la flexión del tronco tocando con las manos juntas en la tapa superior de un cajón (ver Figura 2). Hay ciertas modificaciones de esta prueba, aunque todas poseen la misma validez ${ }^{13,14}$.

Según el procedimiento habitual $^{15}$, se pide al sujeto que realice tres intentos de flexión del tronco llegando todo lo que pueda adelante, tocando en la tapa superior del cajón y manteniendo la posición 2 segundos, anotando el resultado de la mayor flexión conseguida. En todo momento debe mantener la extensión de las rodillas, así como el contacto de las plantas de los pies con el cajón.

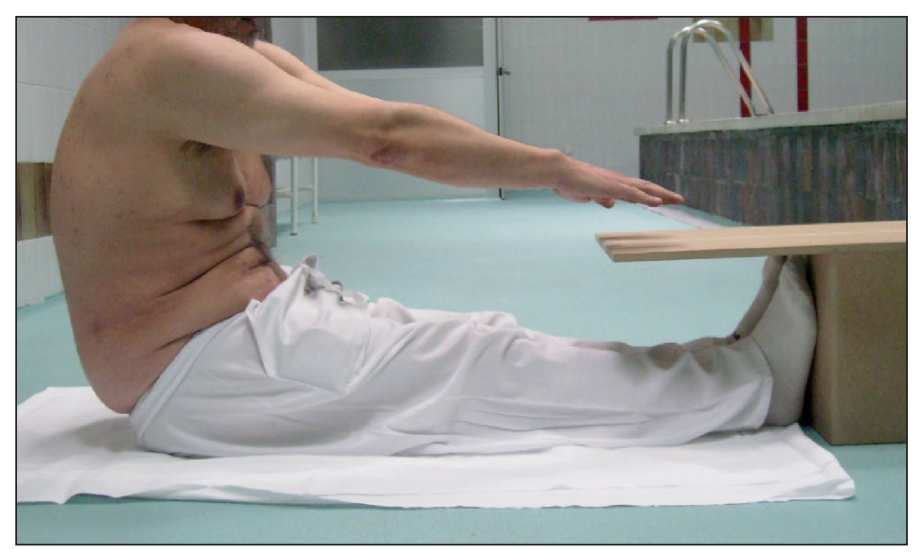

Figura 2. Posición en la prueba sit-and-reach.

En el presente estudio, y tras esta medición inicial, cada sujeto pasa a otra sala donde un fisioterapeuta le aplica el vendaje según la distribución aleatorizada previamente (VNM, esparadrapo o Omniplaste ${ }^{\circledR}-E$ ). A continuación, el sujeto se viste de nuevo. Pasados 15 minutos, se vuelve a realizar la medición en el cajón; al tener puesta una camiseta encima se garantiza el ciego del evaluador que anota la segunda medición SAR. Esta prueba ofrece una medición del alcance global de todo el tronco y hay que considerar cierto margen de error debido a cambios en la flexibilidad de los miembros superiores o en el decremento del ángulo coxofemoral. Para prevenir la distorsión del valor real, en el primer caso se aconseja que los participantes no realicen ejercicios o movimientos amplios con los brazos durante los 15 minutos siguientes a la aplicación de la técnica asignada, aunque pueden moverse. En cuanto a la posible influencia del decremen- 
to del ángulo coxofemoral, se tiene en cuenta a otros investigadores que lo señalan como la causa principal de la ganancia de flexibilidad ${ }^{16}$. Para ello, se recomienda a los sujetos que eviten caminar mucho o de forma rápida, ni tampoco estiren los isquiosurales entre las dos mediciones.

El test de Schober se realiza en dos momentos: justo antes de la aplicación del vendaje, y de nuevo pasados 15 minutos. Según el procedimiento descrito por Buckup ${ }^{17}$, se sitúa al sujeto en bipedestación y se localiza la apófisis espinosa de $\mathrm{S} 1$, donde se hace una pequeña marca con un lápiz dermográfico. Con ayuda de una cinta métrica, se vuelve a marcar otro punto $10 \mathrm{~cm}$ más arriba, y a continuación el sujeto realiza la flexión anterior de tronco (manteniendo las rodillas extendidas), anotando la medida entre los dos puntos, situando de nuevo la cinta métrica a lo largo de la línea de las apófisis espinosas. Posteriormente, sus resultados se analizan y comparan con los proporcionados por la prueba SAR.

\section{Aplicación del vendaje}

Todos los vendajes se realizan siguiendo el mismo procedimiento, que consiste en los siguientes pasos:

1. Sujeto en sedestación con el tronco descubierto, se pegan los primeros $3 \mathrm{~cm}$ de cada tira adhesiva en las proyecciones de la musculatura paravertebral a nivel de $\mathrm{S} 1$.

2. Se pide una ligera flexión de tronco, y a continuación se pega cada venda sin tensión $(0 \%)$ hasta llegar al nivel de T10, a lo largo de la musculatura paravertebral.

3. Se pasan las manos varias veces sobre las tiras para calentarlas (el vendaje neuromuscular es termoplástico; esta maniobra también se realizó con el Omniplaste $^{\circledR}-E$ y el esparadrapo).

4. Se solicita una extensión máxima del tronco contra una ligera resistencia.

Se puede observar la disposición del vendaje aplicado en la zona lumbar, tal y como se muestra en la Figura 3.

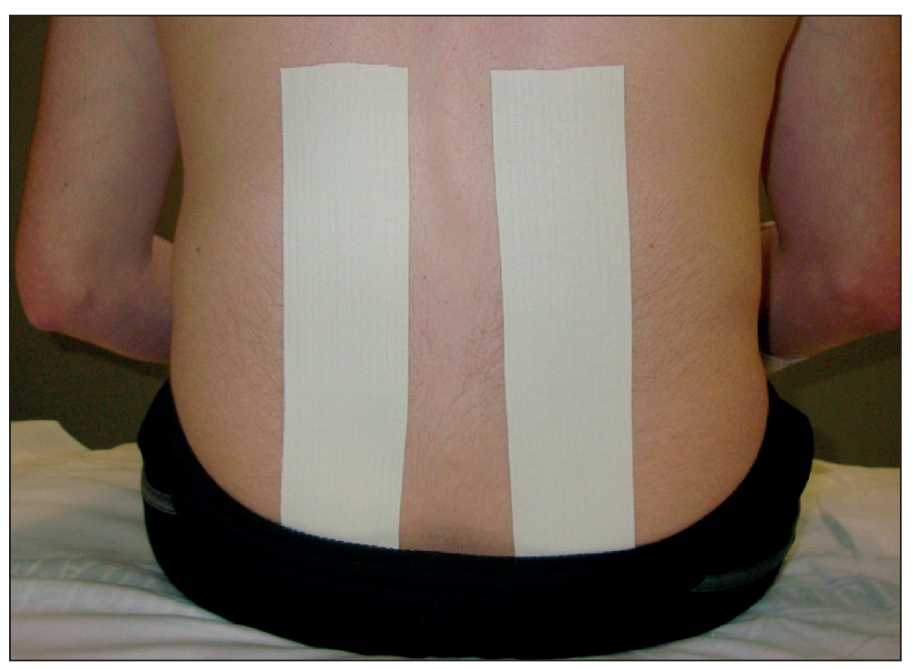

Figura 3. Disposición del vendaje aplicado.

\section{Análisis estadístico}

Se evaluaron las diferencias de los alcances logrados antes y después de la aplicación de cada uno de los tipos de vendaje (VNM, esparadrapo y Omniplaste $^{\circledR}-E$ ) con las pruebas SAR y Schober. Para ello se utilizaron las medianas y los rangos intercuartílicos, empleando para su análisis el test de Wilcoxon, al tratarse de distribuciones no paramétricas declaradas mediante el test de Shapiro-Wilk.

\section{Resultados}

A todos los individuos valorados inicialmente se les aplicó la intervención y completaron las valoraciones post-intervención. Con el fin de evaluar los efectos del VNM sobre la flexión del raquis lumbar, se tuvieron en cuenta dos mediciones. Por un lado, se comparó el valor SAR antes y después de la aplicación del VNM o alguna de las técnicas placebo, y por otro lado se comparó de igual manera la medida obtenida según el test de Schober, en cada uno de los tres grupos. En la Tabla 2 se disponen los resultados numéricos en sendas pruebas, expresados en medianas y rangos intercuartílicos al no darse el supuesto de normalidad de las variables tras haber aplicado el test Shapiro-Wilk.

Tabla 2. Resultados de la prueba SAR y test de Schober.

\begin{tabular}{|c|c|c|c|c|}
\hline & Técnica & Basal (cm) & Final $(\mathrm{cm})$ & $\mathbf{p}^{*}$ \\
\hline \multirow{3}{*}{ Sit-and-reach } & 1 - Esparadrapo & $24(15)$ & $25(13)$ & 0,005 \\
\hline & 2 - Omniplaste ${ }^{\circledR}-\mathrm{E}$ & $26(7)$ & $27(9)$ & 0,018 \\
\hline & $3-\mathrm{VNM}$ & $26,5(13,5)$ & $28(13)$ & 0,011 \\
\hline \multirow{3}{*}{ Schober } & 1 - Esparadrapo & $15(1,5)$ & $15(1)$ & 1 \\
\hline & 2 - Omniplaste $\AA-E$ & $15(1)$ & $15(1)$ & 0,177 \\
\hline & $3-\mathrm{VNM}$ & $14,5(1)$ & $15,5(1,5)$ & 0,001 \\
\hline
\end{tabular}

*Test Wilcoxon

Aunque según la prueba SAR las tres técnicas lograron un incremento de flexión estadísticamente significativo, la diferencia de medianas de flexión basal y final del grupo tratado con VNM fue ligeramente superior que la de los otros dos grupos $(1,5 \mathrm{~cm}$ con VNM frente a $1 \mathrm{~cm}$ con espadrapo u Omniplaste $^{\circledR}-E$ ). Para el test de Schober el comportamiento de las tres técnicas fue diferente, ya que solamente el VNM se mostró eficiente incrementando el valor basal en $1 \mathrm{~cm}$ de mediana $(\mathrm{p}=0,001)$, como ilustra a continuación la Figura 4:

Como se puede observar en la Figura 4, en el caso del esparadrapo y el Omniplaste $^{\circledR}-E$ no se obtuvo incremento alguno de la flexión lumbar según el test de Schober, lo cual induce a pensar que dicho incremento se debe al efecto placebo, mientras que en el caso del VNM sí existe una correlación entre el incremento del alcance en la prueba SAR y el test de Schober. Aunque los valores del test de Schober son mucho menores que los de la prueba SAR, su variación es bastante significativa puesto que éstos se refieren exclusivamente a lo sucedido en la columna lumbar.

Al observarse una discordancia en los resultados del esparadrapo y el Omniplaste $^{\circledR}-E$, ya que según la prueba SAR sus resul- 


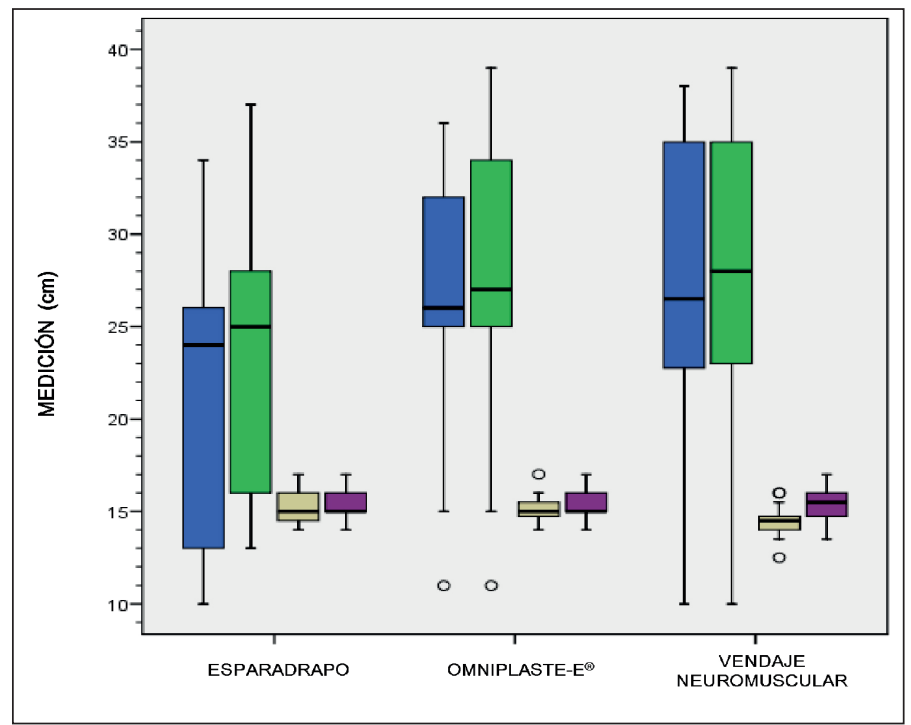

Figura 4. Grupos y medianas de alcance en las pruebas sit-andreach y Schober.

Valor inicial sit-and-reach; Valor sit-and-reach tras intervención; Valor inicial Schober; Valor Schober tras intervención; — Valor de la mediana

tados son estadísticamente significativos y según Schober no, se estudió el incremento de flexión en forma de porcentaje relativo en lugar de diferencia de distancias medidas, obteniéndose los resultados expuestos en la Tabla 3.

Como se aprecia en la Tabla 3, la dispersión de los resultados de la prueba SAR es muy notable, de manera que su medición puede no ser fiable para obtener datos precisos. Esto llega hasta el punto de que se obtiene mayor porcentaje de incremento de flexión con el esparadrapo rígido convencional que con la venda adhesiva elástica, lo cual es incongruente ya que teóricamente el primero debería limitar más el movimiento. Además, no son resultados estadísticamente significativos $(p=0,141)$. La representación gráfica de esta anomalía queda reflejada en la Figura 5.

Por otro lado, según el test de Schober no se obtuvo un aumento de flexión para las dos técnicas placebo, mientras que para el VNM se obtuvo un aumento del 6,25\% de flexión lumbar, todas ellas con una baja dispersión de los datos. Posteriormente,

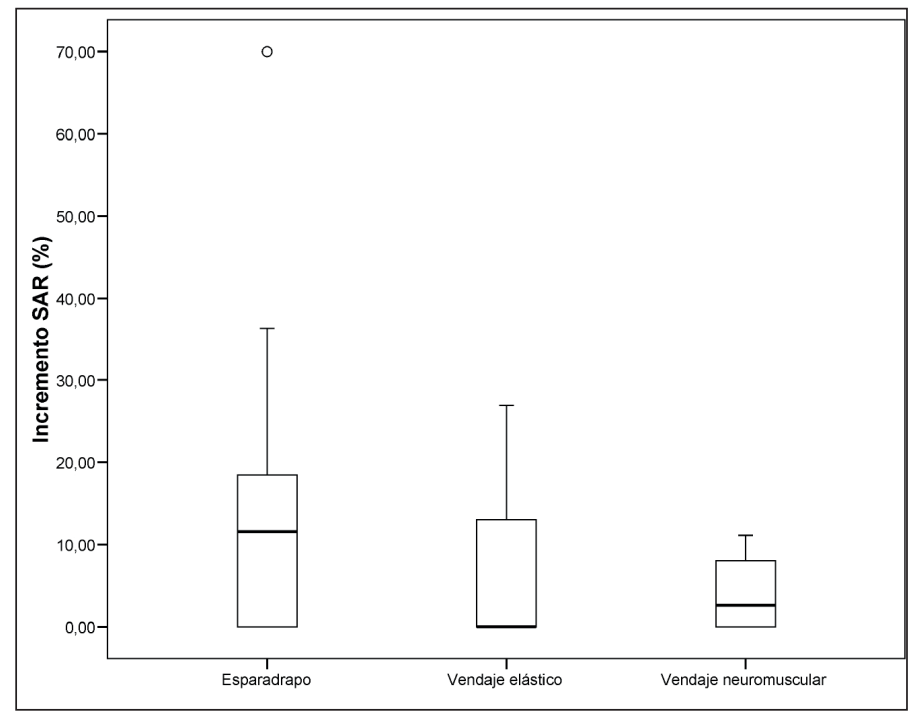

Figura 5. Resultados de la prueba sit-and-reach (en \% relativo). - Valor de la mediana

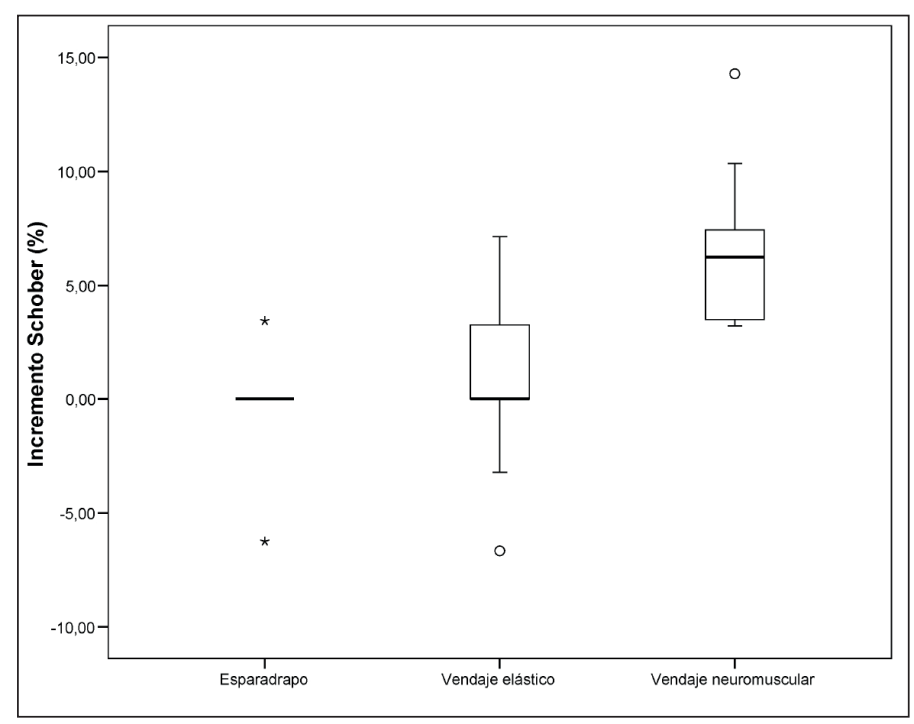

Figura 6. Resultados del test de Schober (en\% relativo).

- Valor de la mediana

Omniplaste $^{\circledR}-E$ y el VNM ( $\left.<<0,001\right)$, como puede observarse en la Figura 6.

Estos resultados muestran por un lado que la prueba SAR no es fiable en la medición de la flexión lumbar, y por otra parte que el VNM logra un significativo porcentaje de incremento de dicha flexión, mayor que el obtenido con las técnicas placebo.

\section{DISCUSIÓN}

Según los resultados expuestos anteriormente, parece evidente que el VNM se muestra como una técnica eficaz en la mejora de la flexión lumbar a corto plazo frente a técnicas placebo como el esparadrapo convencional o el Omniplaste $^{\circledR}-E$, con diferencias estadísticamente significativas. 
También se puede afirmar que, en el caso del VNM, los incrementos de alcance obtenidos en la prueba SAR están relacionados directamente con los valores proporcionados por el test de Schober, de manera que puede establecerse que si bien el aumento del alcance global del tronco no tiene por qué deberse a una mayor flexión lumbar en el caso de las técnicas placebo, sí existe una clara significación estadística para el VNM ( $\mathrm{p}=0,001)$. Estos resultados apoyan los obtenidos por Yoshida y col ${ }^{18}$ quienes afirman que el VNM incrementa la flexibilidad del tronco por su efecto sobre la flexión del mismo. Del mismo modo contradicen lo expuesto por Salvat y coll $^{16}$, quienes sostienen que la causa principal del aumento de la flexibilidad global del tronco es el decremento del ángulo coxofemoral y no una mayor flexión lumbar. Quizás algunas causas de esta discordancia pueden consistir en que Salvat aplica un 10\% de tensión en el VNM en lugar del $0 \%$ de este estudio, y a que muchos datos son obtenidos mediante un software de análisis fotográfico a diferencia de las mediciones directas realizadas in situ en este trabajo.

En consecuencia, se deduce que el VNM salva el efecto placebo causado por la sensación de tener una vendaje aplicado sobre la piel, proporcionando un estímulo sobre diferentes receptores propioceptivos y un efecto mecánico sobre la fascia, viéndose facilitados en primer lugar por la similitud que presenta la venda elástica respecto a la piel, y en segundo lugar por la disposición especial que el fisioterapeuta aplique en cada caso. Aunque los resultados SAR parecen adecuados para el esparadrapo conven-

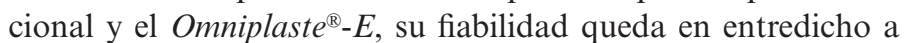
tenor de los datos estudiados como medianas de porcentaje de incremento de flexión. Aunque no se puede descartar del todo un cierto efecto placebo, resulta mucho más fiable el análisis de los datos obtenidos con el test de Schober, en el cual el incremento de la flexión lumbar fue inexistente. La acción terapéutica del VNM es mucho mayor cuando la semejanza de la venda y la piel es máxima, es decir, cuando se imprime una tensión al $0 \%$ al VNM, cuyo efecto no se había medido en otro trabajo hasta ahora.

Por otra parte, cabe destacar que se ha aplicado una atención especial en la descripción y aplicación de la metodología, ya que con frecuencia los autores no explican con detalle el tipo de vendaje que aplican. Yoshida y col sí muestran el vendaje aplicado ${ }^{18}$, pero no trabajan con un grupo placebo, los evaluadores no están cegados respecto al grupo que están estudiando y miden a los participantes en bipedestación, cuando en general la medición suele hacerse con el sujeto en sedestación. Si bien encuentran buenos resultados sobre el dolor lumbar y un aumento muy significativo de la flexión del tronco (más de $15 \mathrm{~cm}$ de variación en la prueba SAR frente a los 1-1,5 cm obtenidos en este estudio), no se puede asegurar que el aumento de ésta última se deba a lo sucedido en la columna lumbar ni incluso a la propia utilización del VNM, fundamentalmente por importantes carencias metodológicas que el presente estudio evita satisfactoriamente.

Ebbers subsana también algunas de estas deficiencias ${ }^{12}$, aunque aplica además el VNM a lo largo del recorrido del nervio ciático. De esta manera, el efecto del VNM podría actuar también indirectamente en la musculatura isquiosural, distorsionando el resultado en el alcance global del tronco, al relajar parte de la cadena muscular posterior a la altura de pelvis y miembro inferior, lo que disminuiría el ángulo coxofemoral. Es decir, se logró un alcance mayor en la prueba SAR, pero debido más a lo sucedido en pelvis y miembro inferior que en la columna lumbar. Aún así, tanto Ebbers como Salvat obtienen unos valores similares a los de este estudio, muy inferiores a los publicados por Yoshida.

Para comprobar si estos efectos podían generalizarse a personas de cualquier edad, se optó por dirigir el estudio a una muestra con un espectro muy amplio situado entre 20 y 55 años, lo cual tampoco se ha realizado nunca hasta la fecha, ya que con frecuencia otros trabajos han sido dirigidos a grupos de edades variables, pero casi siempre entre los 10 y 23 años de edad ${ }^{12,16,18,19}$. En principio lo importante consiste en ver la diferencia del alcance, no el valor del alcance en sí mismo; además, se quiso observar si la edad podía también afectar a la propia variación del dato, como elemento diferenciador respecto a otras publicaciones. Según nuestros hallazgos, confirmamos que la edad no parece ser un elemento diferenciador de la respuesta obtenida en la aplicación del VNM, por lo que su aplicación no debería ir dirigida a una edad de población específica.

\section{CONCLUSIONES}

Se ha demostrado que la flexión de la columna lumbar se incrementa con la aplicación de VNM al 0\% de tensión. Por otro lado, el test de Schober se presenta como una herramienta de medición más fiable y precisa que la prueba SAR en este tipo de estudios, por lo que recomendamos su utilización en investigaciones futuras. Es necesario continuar investigando la efectividad del VNM, mejorando en los aspectos metodológicos con el fin de superar las limitaciones y posibles sesgos de este trabajo para obtener resultados más precisos y fiables.

La aplicación del VNM es una técnica fiable y sencilla de aplicar en patología del aparato locomotor. Dada la alta incidencia y prevalencia de dicha patología en la FAS, debería introducirse en los programas de formación del Cuerpo de Sanidad Militar

\section{AGRADECIMIENTOS}

Los autores agradecen la colaboración del personal del Hospital Central de la Defensa (Madrid) que se prestó voluntario a participar en este estudio.

\section{BIBLIOGRAFÍA}

1. Kase K. Illustrated Kinesio-Taping, 4a ed. Tokyo: Ken'i-Kai; 1994.

2. Tsai CT, Chang WD, Lee JP. Effects of short-term treatment with kinesiotaping for plantar fasciitis. Journal of Musculoskeletal Pain. 2010;18:71-80.

3. González-Iglesias J, Fernández-de-las-Peñas C, Cleland JA, Huijbregts P, Del Rosario Gutiérrez-Vega M. Short-term effects of cervical kinesio taping on pain and cervical range of motion in patients with acute whiplash injury:a randomized clinical trial. Journal of Orthopaedic \& Sports Physical Therapy. 2009;39:515-21

4. Kase K, Wallis J, Kase T. Clinical therapeutic applications of the Kinesio Taping method. Alburquerque NM: Kinesio Taping Association; 2003.

5. Gwang K. Medical Taping Concept: Medical Taping - Cross Taping - Spiral Taping. Enschede: Fisiotape BV; 2005. 


\section{Efectos del vendaje neuromuscular sobre la flexibilidad del raquis lumbar}

6. Kase K, Hashimoto T, Okane T. Kinesio taping perfect manual: Amazing taping therapy to eliminate pain and muscle disorders. Tokyo: Ken'i-Kai Information; 1996.

7. Halseth T, McChesney J, De Beliso M, Vaughn R, Lien J. The effects of Kine$\operatorname{sio}^{\mathrm{tm}}$ Taping on proprioception at the ankle. JSSM. 2004; 3: 1-7.

8. Kapandji A. Cuadernos de fisiología articular. Tomo 3: Tronco y raquis, $5^{\mathrm{a}}$ ed. Madrid: Editorial Médica Panamericana; 1998.

9. Merino R, Mayorga D, Fernández E, Torres-Luque G. Effect of kinesio taping on hip and lower trunk range of motion in triathletes. A pilot study. Journal of Sport and Health Research. 2010;2(2):109-18.

10. Espejo L, Apolo MD. Revisión bibliográfica de la efectividad del kinesiotaping. Rehabilitación. 2011;45(2):148-58.

11. Wells KF, Dillon EK. The sit and reach: a test of back and leg flexibility. Res Quart. 1952; 23: 115-8.

12. Ebbers J, Pijnappel H. La influencia del vendaje neuromuscular en la prueba «Sit and Reach». Spormassage International. 2006; 8: 1-6.
13. Lemmink KA, Kemper HC, De Gref MH, Rispens P, Stevens M. The validity of the sit-and-reach test and the modified sit-and-reach test in middle aged to older men and women. Res Q Exerc Sport. 2003; 74: 331-6.

14. Sai-Chuen S. Validity of the modified back-saver sit-and-reach test: a comparison with other protocols. Med Sci Sports Exerc. 2000; 32: 1655-9.

15. Topendsports.com. The sport \& science resource 2013 [en línea]. Fitness testing [consultado 21/11/2012]. En: http://www.topendsports.com/testing/tests/ sit\&reach.htm.

16. Salvat I, Alonso A. Efectos inmediatos del kinesio taping en la flexión lumbar. Fisioterapia. 2010; 32(2): 57-65.

17. Buckup K. Pruebas clínicas para patología ósea, articular y muscular. Exploraciones-Signos-Síntomas, $2^{\mathrm{a}}$ ed. Barcelona: Masson; 2002.

18. Yoshida A, Kahanov L. The effect of kinesio taping on lower trunk range of motions. Res Sports Med. 2007; 15: 103-12.

19. Kendall FP, Kendall E, Provance PG. Kendall's: músculos, pruebas, funciones y dolor postural, $4^{\mathrm{a}}$ ed. Barcelona: Marban; 2000. 